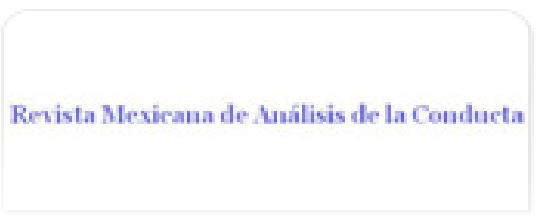

Revista Mexicana de Análisis de la Conducta ISSN: 0185-4534

editora@rmac-mx.org

Sociedad Mexicana de Análisis de la Conducta

México

MARTÍNEZ SÁNCHEZ, HÉCTOR; URZUA BARRIENTOS, RAYMUNDO INDUCCIÓN A BEBER ALCOHOL Y RESTRICCIÓN DE COMIDA: EFECTOS SOBRE COMER, BEBER Y PESO CORPORAL EN RATAS

Revista Mexicana de Análisis de la Conducta, vol. 39, núm. 3, diciembre-, 2013, pp. 15-38 Sociedad Mexicana de Análisis de la Conducta Distrito Federal, México

Disponible en: http://www.redalyc.org/articulo.oa?id=59335809002

- Cómo citar el artículo

- Número completo

- Más información del artículo

- Página de la revista en redalyc.org

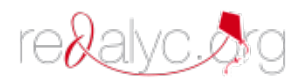

Sistema de Información Científica Red de Revistas Científicas de América Latina, el Caribe, España y Portugal Proyecto académico sin fines de lucro, desarrollado bajo la iniciativa de acceso abierto 


\title{
INDUCCIÓN A BEBER ALCOHOL Y RESTRICCIÓN DE COMIDA: EFECTOS SOBRE COMER, BEBER Y PESO CORPORAL EN RATAS
}

\author{
ALCOHOL DRINKING INDUCTION AND RESTRICTION OF FOOD: \\ EFFECTS ON EATING, DRINKING AND BODY WEIGHT IN RATS
}

\section{HÉCTOR MARTÍNEZ SÁNCHEZ Y RAYMUNDO URZUA BARRIENTOS INSTITUTO DE NEUROCIENCIAS UNIVERSIDAD DE GUADALAJARA-MÉXICO}

\begin{abstract}
Resumen
Se exploraron los efectos de distintos procedimientos de inducción al alcohol y ciclos posteriores de privación-acceso libre a la comida en ratas sobre el mantenimiento del consumo de alcohol, alimento, agua y el peso corporal cuando el alcohol se encontraba disponible. Grupos de ratas con acceso libre a la comida recibieron un procedimiento de inducción para beber alcohol durante 12 días : a) alcohol forzado gradual $(4 \%-10 \% \mathrm{v} / \mathrm{v})$; b) alcohol forzado $(10 \% \mathrm{v} / \mathrm{v} /) ; \mathrm{c})$ agua y alcohol $(6 \%-10 \% \mathrm{v} / \mathrm{v})$ sólo durante la inducción; o, d) el mismo procedimiento anterior pero mantenido durante todo el experimento. D espués de la inducción todos los grupos continuaron con diez días de acceso libre a la comida y alcohol seguido por dos ciclos alternando tres días de restricción total de alimento por diez días de libre acceso a la comida y el alcohol ( $10 \% \mathrm{v} / \mathrm{v})$ siempre estuvo disponible para todos los grupos. Se registró diariamente el peso corporal, los consumos de comida, agua y alcohol. Los resultados

Héctor M artínez Sánchez y Raymundo U rzua Barrientos, Instituto de Neurociencias, Universidad de Guadalajara-M éxico.

Esta investigación se llevó a cabo gracias al apoyo parcial del proyecto 06-2009-542 del Consejo Estatal de Ciencia y Tecnología (CO ECyTJAL). Los autores desean agradecer a la Editora General y a dos revisores anónimos por sus valiosos comentarios y a Eder Espinoza y David Herrera por sus comentarios a versiones tempranas del manuscrito.

Toda correspondencia dirigirla a: Héctor M artínez, Francisco de Q uevedo \#180, Arcos Vallarta, 44100, Guadalajara, Jal. M éxico. Correo electrónico: hectorm@cencar.udg.mx
\end{abstract}


mostraron efectos diferenciales durante la inducción al alcohol sobre el peso corporal y el consumo de alcohol. Posterior a la inducción, para tres de los grupos el consumo de alimento fue estable y sólo para el grupo que tuvo alcohol y agua todo el tiempo se observó un aumento en su consumo de alimento y menor consumo de alcohol en los periodos de acceso libre a comida. Este grupo mostró autoprivación de agua y grandes bebidas de agua post-privación de alimento. Se discute la interacción entre comer, beber y el peso corporal bajo condiciones de restricción de alimento y la disponibilidad de alcohol.

Palabras clave: inducción, alcohol, restricción de comida, comer, beber, peso corporal, ratas

\begin{abstract}
Different methods were explored to induce alcohol intake in rats and subsequent cycles of food deprivation on the consumption of alcohol, food, water, and body weight when alcohol was available. Rats were assigned to four groups and a different procedure to induce alcohol intake was used over 12 days a) gradual forced alcohol $(4 \%-10 \%$ v / v); b) forced alcohol $(10 \%$ v / v /); c) water and alcohol $(6 \%-10 \%$ v / v) only during the induction period; or, d) water and alcohol $(6 \%-10 \%$ v / v) throughout the experiment. After the induction period all groups of rats had free access to food and alcohol for ten subsequent days, followed by two alternating cycles of three days of total food restriction and then ten days of free access to food. After the induction period, alcohol $(10 \% \mathrm{v} / \mathrm{v})$ always was available for all groups. Body weight, food and fluid intake were recorded daily. The induction procedures had differential effects on body weight and alcohol intake. For the rats that had water and alcohol available throughout the experiment, food intake increased and alcohol intake decreased during the days in which food was freely available. A "self-imposed" water deprivation was observed for this group during the food restriction period, and binge drinking occurred when food was available again. The interaction between eating, drinking and body weight under conditions of food restriction and alcohol availability is discussed.

Keywords: induction, alcohol, food restriction, eating and drinking patterns, body weight, rats

Una de las dificultades que enfrentan los investigadores en el estudio de los efectos del consumo de alcohol es mantener un consumo estable de alcohol en sujetos experimentales no humanos. Por lo general las ratas no beben suficientes volúmenes de alcohol voluntariamente y aunque despliegan un aumento gradual tampoco mantienen el incremento en la selección de alcohol en comparación con el agua cuando tienen acceso libre al alcohol (Holloway, Bird, \& Devenport, 1984; Slawecki \& Samson, 1997). Mello (1973) ha referido que a pesar de los esfuerzos que se han
\end{abstract}


realizado en el diseño de técnicas para inducir una preferencia por alcohol en animales, no han conseguido su objetivo debido principalmente a que el sabor del alcohol es aversivo para la mayoría de los animales. Como una solución a esta dificultad, se han desarrollado procedimientos para favorecer la ingesta oral de alcohol para estudiar los efectos de la experiencia de las ratas con alcohol y alcanzar niveles de consumo estables manipulando variables relacionadas con el mantenimiento del consumo de alcohol. M eisch (2001) en una revisión del modelo general de auto-administración oral de drogas en animales ha planteado que la cantidad de estudios que emplean la ruta oral de ingesta de alguna sustancia es menor en comparación con los estudios que hacen uso de la vía de auto-administración intravenosa. Sin embargo, una desventaja de los modelos de auto-administración de alcohol o de otras sustancias por vía intragástrica o intravenosa es que no permiten estudiar los cambios conductuales o fisiológicos graduales que se producen mediante la auto-administración oral (Slawecki \& Samson, 1997).

Samson (1986) ha señalado que se ha tenido poco éxito para inducir a beber alcohol por la ruta oral a ratas, a menos que se priven de comida y/o líquidos para iniciar y mantener la auto-administración de alcohol. Richter (1926) mostró que cuando las ratas se han adaptado a beber agua tras un periodo de 25 a 30 días y se les expone al alcohol las ratas muestran un rechazo a beberlo. Slawecki y Samson (1997) han reportado que ratas sin experiencia de beber alcohol no consumen voluntariamente suficientes volúmenes de soluciones de alcohol diluido (>10\%) para producir niveles de alcohol en la sangre que puedan ser asociados con los cambios conductuales.

M eisch (2001) en su revisión identificó tres problemas principales que hacen difícil conseguir que los animales ingieran drogas oralmente: a) el sabor aversivo de las soluciones conteniendo droga y que los animales prefieren agua si tienen la opción; la restricción del acceso al líquido que contiene droga puede producir la ingesta de la droga. Sin embargo, en el caso de los estudios operantes la restricción de la droga no siempre la convierte en un reforzador efectivo; b) animales sin experiencia en beber una solución con droga ingieren cantidades muy pequeñas de la solución dificultando la evaluación de los efectos producidos por el consumo de la droga; y, c) el intervalo entre la conducta de beber la solución con droga y el efecto subsecuente en el sistema nervioso central toma más de 5 minutos dificultando las posibilidades de su empleo como reforzador en los estudios de condicionamiento operante.

De acuerdo con Koob (2000) hay varios modelos de auto-administración oral para producir beber alcohol en animales. U no de ellos es la privación de alcohol en el que los animales son sometidos a una abstinencia forzada de alcohol después de haberlo consumido regularmente y los animales lo beben excesivamente cuando el alcohol vuelve a estar disponible (Sinclair \& Senter, 1967). H eyser, Schulteis y Koob (1997) han señalado que en el estudio de los parámetros temporales de la disponibilidad de alcohol se descubrió que una característica confiable y robusta observada en los 
modelos animales de beber alcohol, es un incremento en el consumo que ocurre después de un periodo de privación de alcohol. Estos autores añaden que este efecto se ha reportado en ratones, ratas, monos y bebedores sociales humanos y ha sido denominado el efecto por privación de alcohol. Bell, Rodd, Lumeng, Murphy, y M cBride (2006) empleando ratas criadas con preferencia por el alcohol para estudiar los mecanismos que participan en la recaidas de beber alcohol, después de que las ratas desarrollaron un consumo de alcohol crónico utilizaron periodos repetidos de privación de alcohol encontrando que dos o más privaciones, junto con el acceso concurrente a múltiples concentraciones de alcohol después de cada privación, produjeron un pronunciado incremento en el consumo de alcohol cuando lo tienen nuevamente disponible.

Los procedimientos de privación se han usado extensamente para facilitar la conducta de consumo que está relacionada con la sustancia que ha sido retirada (e.g., alcohol) y bajo ciertas circunstancias, produce un aumento temporal en el consumo de tal sustancia, como sucede con los reforzadores naturales como la comida y el agua (Heyser, Schulteis, \& Koob, 1997). La disponibilidad o no de agua adicional al alcohol durante la inducción de alcohol en ratas, ha producido diferencias significativas en el aumento del patrón de consumo de alcohol posterior a la inducción cuando el agua estuvo disponible (Veale \& M yers, 1969). Esto podría sugerir que cuando las ratas son inducidas al alcohol con agua adicional disponible beberán más alcohol comparadas con una inducción de alcohol forzado (para una revisión en extenso ver Cunningham, Fidler, \& Hill, 2000).

Holloway, Bird y Devenport (1984) especificaron al menos cinco factores que pueden influir en el grado de selección del alcohol en ratas: a) la concentración de alcohol; b) la naturaleza de la experiencia previa con alcohol; c) la frecuencia de acceso al alcohol; d) la regularidad del acceso periódico al alcohol; y, e) diferencias en la responsividad al programa variable. Holloway et al. (1984) denominaron efecto de disponibilidad periódica al fenómeno que ocurre cuando a ratas se les da la oportunidad periódica para consumir alcohol produciendo un incremento gradual y confiable en la selección de alcohol cuando se les prueba con agua como opción. Sin embargo, la preferencia por el alcohol no se sostiene cuando el alcohol está disponible de forma continua. Este fenómeno tiene características similares a los efectos producidos por la privación de alcohol con ratas en el que también ocurre un aumento temporal en la selección de alcohol después del retiro de un régimen de consumo crónico de alcohol. Estas formas de inducción al alcohol producen aumentos temporales pero no sostenidos de la preferencia del alcohol cuando el acceso al alcohol está libremente disponible.

En los modelos de auto-administración oral los sujetos controlan la ingesta de alcohol y se ha mostrado que la cantidad de alcohol que consumen puede depender de si su disponibilidad es precedida o no por un procedimiento de inducción. Cunningham, Fidler y Hill, (2000) han señalado que en los modelos de auto-administración los animales controlan su ingesta de alcohol determinando la cantidad consumida y el 
patrón temporal de consumo, distinguiendo así los modelos de auto-administración de beber en la caja-hogar de los de condicionamiento operante, en los que el experimentador controla la dosis cada vez que se administra el alcohol (e.g., como reforzador). Para el experimentador un procedimiento de inducción bajo un modelo de auto-administración representa la introducción controlada del alcohol en la dieta y patrones de consumo de un organismo (e.g., dosis, disponibilidad). Se ha documentado que en pruebas de preferencia las ratas beben alcohol únicamente en dosis muy bajas comparadas con beber agua (M eisch, 1984; M ello \& M endelson, 1964). Kahn y Stellar (1960) han mostrado que las dosis iniciales y la experiencia con alcohol en ratas están relacionados con el nivel de preferencia por el alcohol. Con dosis muy bajas muestran preferencia, con intermedias indiferencia y con dosis altas el alcohol se vuelve aversivo.

Slawecki y Samson (1997) han planteado la hipótesis del sabor adquirido del alcohol que es apoyada por el aumento en el consumo de alcohol y la preferencia del alcohol en su caja-hogar después del procedimiento de sustitución de sacarosa. Los diversos procedimientos para inducir beber alcohol a ratas habitualmente incluyen la técnica de adulteración de una solución de alcohol con sacarosa o con otras sustancias dulces para mantener la conducta reforzada con alcohol (M eisch, 2001). Un problema con esta técnica es que cuando la sustancia dulce es retirada se mantiene muy débil la conducta reforzada por alcohol. Por lo general, el retiro de la sustancia endulzante no es gradual, se hace sin tomar en cuenta la relación entre la concentración de la sustancia endulzante y la concentración de alcohol. Samson (1986) asumió que debido al súbito retiro de la sustancia dulce se producía el pobre mantenimiento de la conducta reforzada por alcohol. Samson (1986) utilizó un procedimientro de iniciación de alcohol diluido en una solución endulzada sin privación de comida o agua para establecer el alcohol como un reforzador administrado oralmente. Con ese procedimiento demostró que introducir una solución endulzada (e.g., sacarina) en alcohol y después desvanecer la solución dulce produjo un consumo estable de alcohol en ratas en un periodo de entre 6 y 8 semanas. Heyser et al. (1997) con un procedimiento operante entrenaron a ratas a auto-administrarse alcohol oralmente usando el procedimiento de desvanecimiento de sacarina y comparado con las sesiones de línea base incrementaron la auto-administración de alcohol entre un 30\% y $40 \%$ después de la abstinencia impuesta de alcohol de 5 días o más. Cunningham et al. (2000) han señalado que los animales no ingieren grandes volúmenes de una alta concentración de alcohol diluido (e.g., 30\%) la primera vez que lo tienen disponible y sólo lo prefieren en concentraciones bajas (e.g., 4\%-6\%).

Entre los modelos animales de auto-administración oral empleados para analizar las alteraciones en el comportamiento alimentario se encuentra la privación o restricción de alimento 0 agua (Corwin \& Buda-Levin, 2004; M eisch, 2001). Bajo este modelo cuando grupos de ratas son expuestos a ciclos de privación-acceso a la comida se obtienen como resultado cambios en el peso corporal y el consumo de alimento y 
de agua si se compara con grupos de ratas que no son expuestos a estos ciclos (e.g. Corwin \& Buda-Levin, 2004; M artínez \& Gómez, 2009). Algunos de los cambios que se han identificado en los patrones de consumo de agua o comida bajo estas condiciones han sido reportados como el binge eating o gran comilona en el primer día de acceso al alimento 0 al agua después de la privación de alimento 0 agua (Corwin $\&$ Buda-Levin, 2004; H agan \& M oss, 1997). Verplanck y H ayes (1953) reportaron que durante el periodo de privación de alimento en ratas con acceso libre al agua, se produjo una disminución del consumo de agua comparado con el consumo de agua previo al periodo de privación y durante la privación de agua estando el alimento en acceso libre se produjo una disminución del consumo de alimento. A esta reducción en el consumo durante los periodos de privación es lo que denominaron como autoprivación. $\mathrm{O}$ tros cambios reportados son el beber excesivo post-privación de alimento 0 agua (Siegel $\&$ Talantis, 1948) y un aumento o recuperación del peso corporal posterior a la restricción de alimento (López-Espinoza \& Martínez, 2001, 2004, 2005).

Se ha mostrado que mientras mayor sea el tiempo de restricción de alimento 0 agua, el consumo posterior a la restricción será proporcional al periodo de ausencia del agua o alimento restringido (Siegel \& Talantis, 1948). Diversos estudios también han mostrado los efectos de la modificación del patrón alimentario aumentando el consumo de alimento posterior a la restricción de comida (Hagan \& M oss, 1997; López-Espinoza \& Martínez, 2001, 2005) incluyendo el consumo de agua, aún cuando ésta no haya sido restringida (Dimitriou, Rice, \& Corwin, 2000; Linseman \& Harding, 1989; López-Espinoza \& M artínez, 2001, 2005; Sclafani \& Gorman, 1977) o el consumo de alcohol (Soderpalm \& Hansen, 1999). La restricción de agua 0 comida es considerada una variable motivacional, ya que incrementa los patrones de la búsqueda de agua y alimento en ratas (Bindra, 1947).

Una línea de investigación que ha utilizado el modelo animal de privación de alimento también se ha dirigido al estudio de la relación entre la restricción de comida y el consumo de alcohol. Se ha reportado que cuando un grupo de ratas se encuentra privado de comida (e.g., $80 \%$ de su peso corporal) y en condiciones de libre acceso al alcohol y al agua consumen más alcohol que agua. Incluso, una vez eliminada la restricción de comida y regresando a su peso corporal normal, las ratas continuaron mostrando una preferencia por el alcohol sobre el agua, aunque en menor medida que durante los periodos de privación de comida (M eisch, 1984). También se ha documentado que la privación de alimento produce un incremento de la ingesta de alcohol muy similar al consumo producido por la privación de agua en ratas (Soderpalm \& Hansen, 1999).

En una revisión no exhaustiva, la literatura sobre restricción de comida y consumo de alimento, agua o alcohol muestra que se ha utilizado la reducción del peso al $80 \%$ para inducir un incremento en la ingesta de alcohol (Linseman \& Harding, 1989; \} Soderpalm \& Hansen, 1999), o restricciones parciales de alimento de 12 horas son suficientes para producir una disminución en el consumo de agua durante el periodo de restricción de comida, aún cuando era el único líquido disponible y sin res- 
tricción (López-Espinoza \& Martínez, 2001), o bien, usando fármacos del tipo antagonistas opioides (e.g., naltrexona) en combinación con privación de alimento para evaluar la ingesta de comida y alcohol (Barrios De Tomasi \& Juárez, 2011). LarueAchagiotis, Poussard y Louis-Sylvestre (1990) con el propósito de evaluar si el consumo de alcohol afecta la ganancia de peso corporal en ratas, compararon el peso corporal, el consumo de comida y de agua o alcohol diluido al $10 \%$ ó al $20 \%$ en ratas que recibieron alcohol como única fuente de líquido y en ratas que bebían solo agua. Los grupos de ratas que recibieron agua o alcohol al 10\% no redujeron su ganancia de peso corporal, mientras que las ratas que recibieron alcohol al $20 \%$ redujeron notablemente su ganancia de peso corporal pero mantuvieron sin modificar la cantidad de calorías consumidas vía la ingesta de comida y de alcohol. La importancia de este estudio radica en que el consumo de alcohol al $20 \%$ en ratas causó una marcada reducción en la ganancia de peso corporal sin afectar su consumo de comida.

Grant y Sampson (1985) reportaron que el procedimiento de inducción al alcohol que utilizaron fue efectivo para obtener auto-administración oral de alcohol en cantidades suficientes para producir intoxicación en ratas Long Evans. En su procedimiento de inducción sin privación de alimento, las ratas fueron reforzadas con una solución de sacarosa $(20 \%)$ por beber alcohol $(5 \% \mathrm{v} / \mathrm{v})$. Posterior al procedimiento de inducción el alcohol fue utilizado como reforzador por presionar una palanca en un programa de Razón Fija (RF8) y se mantuvieron trabajando aún bajo concentraciones de alcohol que iban del $10 \%$ al $40 \%$. Estos autores concluyeron que su técnica de inducción ofrece ventajas para investigar los posibles factores iniciadores involucrados con el consumo de alcohol excesivo.

Considerando que las experiencias iniciales de las ratas con alcohol pueden ser determinantes de su consumo futuro, nuestro interés se centró en si después de un periodo de inducción para beber alcohol forzado (gradual vs no gradual) o no forzado (con opción de beber agua) bajo un modelo de auto-administración oral en ratas y la posterior exposición a dos ciclos de privación-acceso a la comida teniendo libre acceso al alcohol $(10 \% \mathrm{v} / \mathrm{v})$, se mantiene el consumo de alcohol y produce cambios en la ganancia de peso corporal y en los patrones de consumo de alimento y agua. Estas condiciones nos permitirían evaluar bajo un modelo de auto-administración oral si la inducción al alcohol, sin añadir una sustancia endulzante ni privación de comida durante la inducción, favorece el mantenimiento del consumo de alcohol.

\section{Método}

\section{Sujetos}

D oce ratas hembras Wistar ingenuas de entre 4 y 5 meses de edad (265-320 g) provenientes del bioterio del Instituto de Neurociencias sirvieron como sujetos 
y fueron asignados a uno de cuatro grupos experimentales $(n=3)$, mantenidos en un ciclo 12-12 horas de luz-0scuridad (7:00/19:00) y a una temperatura constante $(23 \pm 2 \div$ C).

\section{Aparatos y materiales}

Se utilizaron 12 cajas-habitación individuales de acrílico transparente $(21 \times 45 \times 24$ $\mathrm{cm})$. La superficie de las cajas tenía una reja metálica con un orificio para el bebedero y un espacio para colocar la comida. Se utilizó una báscula electrónica de precisión de marca comercial para medir el alimento y el peso corporal de los sujetos. La comida consistió en croquetas para roedores de la marca Rodent Laboratory Chow con la fórmula nutricional: $3 \%$ de grasas, $23 \%$ de proteína, $7 \%$ de ceniza, $1 \%$ de calcio, $6 \%$ de fibra, $49 \%$ de extracto libre de nitrógeno (E. L. N.), 6\% de fósforo y $12 \%$ de humedad. El líquido se midió con una probeta graduada y la disponibilidad del alcohol consistió de una solución de $90 \%$ de agua por $10 \%(\mathrm{v} / \mathrm{v})$ de alcohol comercial $\left(98^{\circ}\right)$.

\section{Procedimiento}

Los sujetos vivieron todo el tiempo en cajas-habitación individuales. Se registró el peso corporal, alimento y agua ingerida cada 24 horas durante diez días. Se estableció un periodo de 12 días para el procedimiento de inducción al alcohol con acceso libre a la comida. Después hubieron 10 días de alcohol (10\%) y comida disponible. Al día siguiente iniciaba un periodo en el que el alimento era retirado durante 72 horas. U na vez cumplido el periodo de restricción de alimento se colocaba nuevamente la comida $(50 \mathrm{~g}$ ) en el espacio de la rejilla que se encontraba en la superficie de la caja después de que los sujetos habían sido pesados. Dependiendo del procedimiento de cada grupo podía haber un bebedero con agua, o bien un bebedero con agua y otro con alcohol. Cuando habían dos bebederos diariamente se alternaba su colocación en la rejilla de la caja. Los bebederos contenían $100 \mathrm{ml}$ de agua y los bebederos que contenían la solución con alcohol diluido $90 \mathrm{ml}$ de agua y $10 \mathrm{ml}$ de alcohol (10\% v/v). Los bebederos y comederos eran rellenados diariamente con las cantidades indicadas. La recolección de datos se llevó a cabo diariamente entre las 10:00 y las 11:00 am durante todo el experimento. Se formaron cuatro grupos experimentales que iniciaron el estudio con el procedimiento de inducción al alcohol de la siguiente manera:

Grupo alcohol forzado con inducción (SA). El objetivo con este grupo era evaluar si incrementar gradualmente la cantidad de alcohol produciría un aumento gradual del consumo de alcohol cuando se llegara al límite establecido (10\%). Este procedimiento tuvo una duración de 12 días y lo denominamos de alcohol forzado con inducción porque los sujetos estaban obligados a beber el alcohol sin tener 
acceso a otra opción para consumir líquido. Los sujetos de este grupo tuvieron acceso todo el tiempo a un solo bebedero que contenía una solución de alcohol diluido ( $4 \%-10 \%$ v/v). Los primeros tres días estuvo disponible el alcohol al $4 \%$; los siguientes 3 días se incrementó el alcohol al $6 \%$, seguido de otro periodo igual con $8 \%$ y finalmente tres días al $10 \%$ (ver Tabla 1 ). La comida estuvo disponible durante el procedimiento de inducción. U na vez concluido el procedimiento de inducción inmediatamente inició el primer período de 10 días con acceso libre al alimento y al alcohol al $10 \%$.

Tabla 1

Porcentaje de alcohol suministrado en el procedimiento alcohol forzado con inducción (SA)

\begin{tabular}{cc}
\hline Día & Alcohol \\
\hline $1-3$ & $4 \%$ \\
$4-6$ & $6 \%$ \\
$7-9$ & $8 \%$ \\
$10-12$ & $10 \%$ \\
\hline
\end{tabular}

Grupo alcohol-agua sólo en inducción (AAA). El objetivo con este grupo fue evaluar la inducción al alcohol teniendo la opción de beber agua y la solución de alcohol. Se aumentó gradualmente la concentración de alcohol pero intercalando algunos días con beber alcohol forzado para que los sujetos se familiarizaran con la condición de tener solo una fuente de líquido. Diariamente se colocaron dos bebederos, uno con agua $(100 \mathrm{ml})$ y otro que inició con una concentración de alcohol al $6 \%$. Al cuarto día se colocó sólo un bebedero con la misma graduación de alcohol. Los días 5, 6, 7 y 8 se introdujeron nuevamente dos bebederos, uno con agua y el otro con alcohol (8\%). Al noveno día se dejó únicamente el bebedero con alcohol (8\%). Finalmente, se colocaron otra vez dos bebederos, uno con agua y el otro con alcohol (10\%) durante tres días (ver Tabla 2). Al finalizar el procedimiento de inducción se inició un periodo de diez días con un solo bebedero conteniendo alcohol (10\%) y acceso libre a la comida. En resumen, los dos bebederos con sólo agua y con alcohol diluido estuvieron disponibles únicamente durante el periodo de inducción intercalando dos días de alcohol forzado. Después de doce días de inducción el experimento prosiguió sólo con el bebedero conteniendo la solución de alcohol al $10 \%$. 
Tabla 2

Porcentaje de alcohol y cantidad de agua en mililitros suministrados en el procedimiento alcohol y agua sólo durante la inducción (AAA y AATT)

\begin{tabular}{ccc}
\hline Día & Alcohol & Agua \\
\hline $1-3$ & $6 \%$ & $100 \mathrm{ml}$ \\
4 & $6 \%$ & \\
$5-8$ & $8 \%$ & $100 \mathrm{ml}$ \\
9 & $8 \%$ & \\
$10-12$ & $10 \%$ & $100 \mathrm{ml}$ \\
\hline
\end{tabular}

Grupo alcohol-agua todo el tiempo (AATT). La inducción al alcohol para este grupo fue similar al grupo AAA (Tabla 2). La única diferencia consistió en que mientras al grupo AAA se le suspendió la presentación del bebedero con agua después del periodo de inducción, para el grupo AATT se mantuvieron disponibles tanto el agua como la solución de alcohol (10\%) durante el resto del experimento. Por lo tanto, exceptuando los dos días cuarto y noveno de alcohol forzado en la inducción, este grupo siempre tuvo disponibles los dos bebederos uno con agua y otro con alcohol a Io largo de todas las fases experimentales. El objetivo era evaluar si habría variaciones en el peso corporal y en los patrones de consumo de alcohol, agua y alimento al mantener la disponibilidad de las dos fuentes de bebida (agua y alcohol) después de haber sido expuestos al procedimiento de inducción.

Grupo alcohol forzado sin inducción (AF). Este grupo de sujetos sirvió como control para los otros tres grupos que recibieron el procedimiento de inducción gradual al alcohol. Se mantuvieron los 12 días de inducción de los otros grupos, pero sólo tuvieron un bebedero con alcohol (10\%) desde un inicio (alcohol forzado). Como en todos los grupos anteriores los ciclos de acceso libre y restricción de la comida iniciaron al siguiente día de terminado el periodo de inducción.

Restricción y acceso libre de comida. U na vez completados los 12 días del procedimiento de inducción al alcohol con la comida siempre disponible, se inició un periodo de 10 días en los que también había libre acceso a la comida y a la solución de alcohol ( $10 \% \mathrm{v} / \mathrm{v}$ para el resto del experimento) para todos los grupos y sólo para el grupo AATT también el agua estuvo disponible libremente. Los siguientes tres días se retiró el alimento durante 72 horas consecutivas con el alcohol disponible todo el tiempo para todos los grupos. En seguida iniciaba un nuevo periodo de 10 días de libre acceso a la comida manteniéndose el alcohol disponible. Sólo el grupo AATT tuvo disponible un bebedero de agua además del de alcohol durante todo el 
experimento. El ciclo de restricción-libre acceso de comida se repitió una vez más bajo las mismas condiciones y duración finalizando el experimento al completarse 48 días continuos de observación.

\section{Resultados}

Los datos se presentan en forma grupal debido a que no hubo variaciones individuales importantes dentro de los cuatro grupos. Las Figuras 1, 2 y 3 muestran los promedios diarios y desviación estándar del peso corporal, del consumo de alimento en gramos y del consumo de alcohol en gramos $/$ kilogramos $(\mathrm{g} / \mathrm{kg})$ de cada grupo de sujetos. Los primeros 12 días de cada figura corresponden al periodo de inducción al alcohol. Los siguientes 10 días son de libre acceso a la comida y alcohol. A continuación aparecen los tres días de restricción de comida seguidos por 10 días de libre acceso a la comida y alcohol y posteriormente el ciclo restante de restricción-acceso libre de comida y alcohol con el que finalizó el experimento.

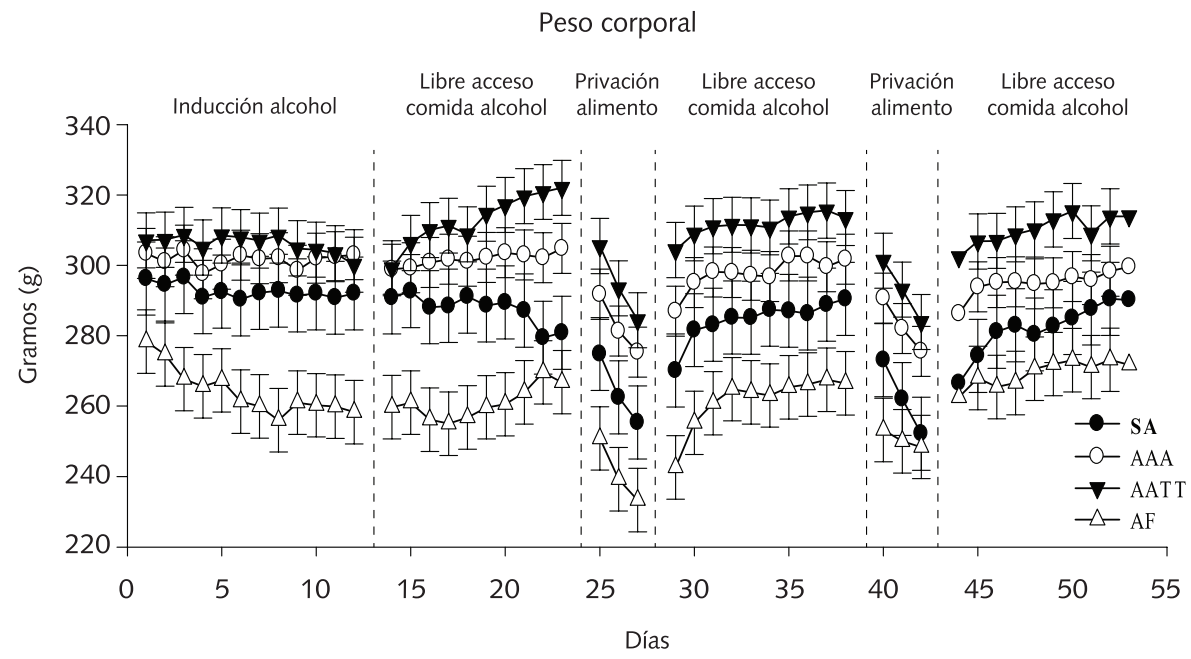

Figura 1. Medias y desviación estándar del peso corporal en gramos de cada uno de los cuatro grupos de sujetos durante el periodo de inducción y a lo largo de las fases experimentales. SA (círculos negros, alcohol con inducción); AAA(círculos blancos, alcohol-agua durante la inducción); AATT (triángulos negros invertidos, alcohol y agua todo el experimento); AF (triángulos blancos, alcohol forzado sin inducción). 


\section{Grupo alcohol forzado con inducción}

El peso corporal de los sujetos de este grupo (círculos negros de la Figura 1) durante todo el periodo de inducción al alcohol se mantuvo estable por debajo de los $300 \mathrm{~g}$ mostrando una ligera disminución hacia el final de la inducción. En el periodo previo a la primera restricción de alimento esta disminución fue mayor en los dos últimos días. Durante la primera restricción de comida todos los sujetos mostraron una disminución gradual del peso corporal hasta aproximadamente los $250 \mathrm{~g}$ en el tercer día. En el primer día de acceso libre a la comida subieron de peso sin alcanzar a recuperar el peso corporal previo a la restricción, lo que ocurrió al final de esta condición. Este patrón se replicó en el siguiente ciclo restricción-acceso libre de comida.

El consumo de alimento (círculos negros de la Figura 2) mostró mayor variabilidad que el peso corporal durante la inducción al alcohol consumiendo un máximo de 18 $\mathrm{g}$ el segundo día y un mínimo de $10 \mathrm{~g}$ en el sexto día de la inducción. No obstante, durante el primer libre acceso a la comida hubo menor variabilidad y un menor consumo de alimento que durante la inducción. Un dato interesante fue el ligero incremento del consumo de alimento posterior a la primera restricción de alimento, aún cuando continuó habiendo variabilidad a lo largo de la condición. En el último acceso libre a la comida, aunque en menor grado, se mantuvo la variabilidad.

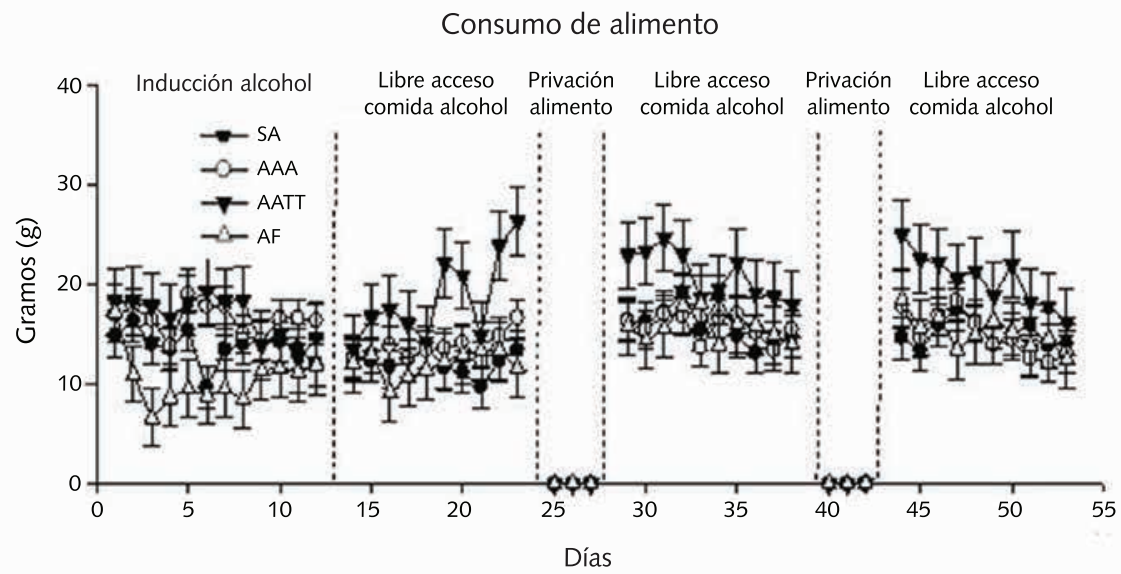

Figura 2. Medias y desviación estándar del consumo de alimento en gramos de cada uno de los cuatro grupos de sujetos. SA (círculos negros, alcohol con inducción); AAA (círculos blancos, alcohol-agua durante la inducción); AATT (triángulos negros invertidos, alcohol y agua todo el experimento); $\mathrm{AF}$ (triángulos blancos, alcohol forzado sin inducción). 
En contraste, el consumo de alcohol mostró alta variabilidad a lo largo de la inducción (círculos negros de la Figura 3). La cantidad ingerida incrementó los primeros tres días hasta rebasar los $60 \mathrm{ml}$, pero luego hubo una disminución constante que llegó a $25 \mathrm{ml}$ en el día once. Durante el primer periodo de acceso libre a la comida se registraron consumos de alcohol alrededor de los $35 \mathrm{ml}$. En el primer día de privación de alimento el consumo de alcohol aumentó con respecto al último día de la condición previa disminuyendo en los dos días siguientes. Durante la primera restricción de alimento el consumo se mantuvo variable pero en un patrón similar al del primer periodo de libre acceso. En la segunda restricción de alimento el consumo de alcohol estuvo por debajo de todas las condiciones anteriores $(<30 \mathrm{ml})$. En la última fase de acceso libre a la comida el consumo de alcohol se recuperó aunque mostró variabilidad.

\section{Grupo agua/alcohol con inducción (AAA)}

Durante el periodo de inducción al alcohol con el bebedero de agua y el de alcohol disponibles, los sujetos de este grupo mantuvieron el peso corporal estable y ligeramente por arriba de los $300 \mathrm{~g}$ (círculos blancos de la Figura 1). En el primer periodo de acceso libre a la comida, sólo con el bebedero con alcohol disponible, hubo un incremento gradual del peso rebasando hacia el final los $300 \mathrm{~g}$. En la primera restricción de comida el peso corporal disminuyó gradualmente durante los tres días hasta llegar a menos de $280 \mathrm{~g}$, seguido por la fase de libre acceso a la comida que dio lugar a una recuperación gradual que alcanzó el peso corporal mostrado en el primer libre acceso. El segundo ciclo de restricción-acceso libre de comida básicamente replicó los resultados del primer ciclo.

En el consumo de alimento (círculos blancos de la Figura 2) se produjo variabilidad a lo largo del periodo de inducción con una reducción en los días cuarto y noveno en los que estaba solo el alcohol disponible. El primer periodo de libre acceso a la comida se mostró un consumo de alimento más estable, aunque hubo una ligera disminución del consumo comparado con la inducción y un incremento al final de la condición. En los periodos posteriores a las restricciones de comida, excepto por sutiles disminuciones hacia el final de ambas condiciones, no ocurrió al gún cambio notorio en el patrón de consumo con respecto al mostrado durante la inducción y al periodo previo a la primera restricción de alimento.

En cambio la ingesta de alcohol sufrió modificaciones en todas las fases del experimento (círculos blancos de la Figura 3). En el periodo de inducción el consumo de alcohol se mostró irregular. El consumo inicial fue el más bajo de todos los grupos en este periodo $(<30 \mathrm{ml})$. En el cuarto día cuando se retiró el agua hubo un aumento en el consumo de alcohol (alcanzando casi $40 \mathrm{ml}$ ) con respecto al consumo del día anterior con agua y alcohol disponibles, disminuyendo en los cuatro días siguientes consumiendo menos de $20 \mathrm{ml}$ de alcohol. En el 


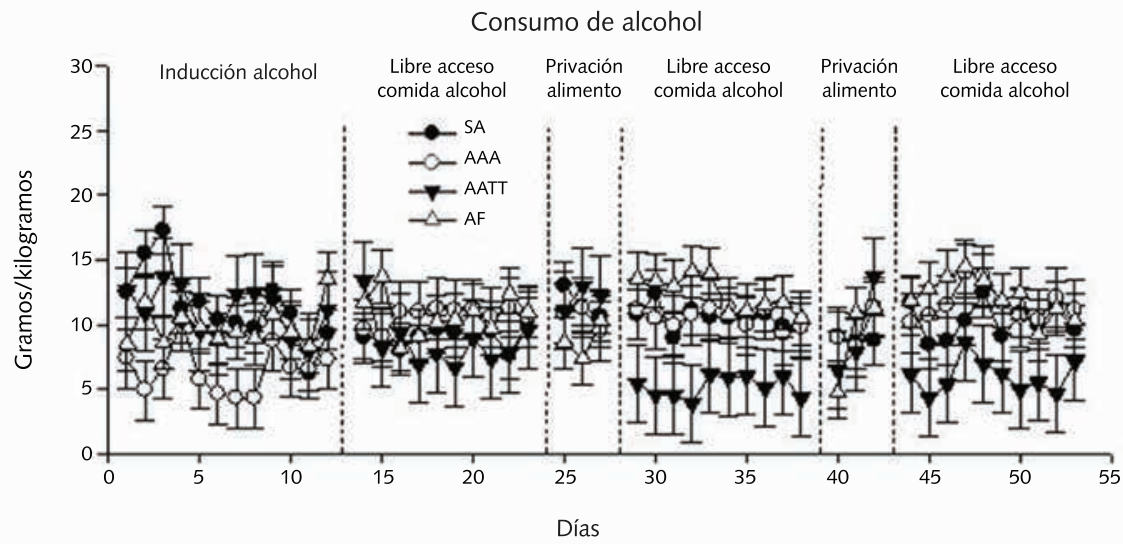

Figura 3. Media y desviación estándar del consumo de alcohol en gramos sobre kilogramos $(\mathrm{g} / \mathrm{kg}$ ) para los cuatro grupos durante el periodo de inducción y a lo largo de todas las fases experimentales. Los círculos negros corresponden el grupo solo alcohol (SA); los círculos blancos al grupo alcohol-agua solo durante la inducción (AAA); los triángulos negros invertidos al grupo alcohol-agua todo el tiempo (AATT); los triángulos blancos al grupo alcohol forzado sin inducción (AF).

noveno día, otra vez sin agua, el consumo de alcohol volvió a incrementar a 35 $\mathrm{ml}$ y en los tres últimos días el consumo se estabilizó alrededor de los $30 \mathrm{ml}$. D urante el primer periodo de libre acceso a la comida el consumo de alcohol se situó por encima del consumo mostrado durante la inducción manteniéndose en ese nivel en el siguiente periodo de libre acceso a la comida $(40 \mathrm{ml})$. D urante las restricciones de alimento el consumo en general no disminuyó como se esperaría si hubiera ocurrido la auto-privación, aunque hubo mayor variabilidad en la segunda restricción.

La columna derecha de la Figura 4 muestra las gráficas individuales del consumo de agua durante el procedimiento de inducción al alcohol. El consumo se mantuvo estable a lo largo del periodo y se consumieron cantidades similares entre sujetos (60 $\mathrm{ml}$ y $70 \mathrm{ml}$ ). Cabe recordar que para este grupo el agua sólo estuvo disponible los primeros doce días de inducción, el resto del experimento únicamente el alcohol estuvo disponible.

\section{Grupo alcohol-agua todo el tiempo (AATT)}

Este grupo con un bebedero de agua y otro con alcohol disponibles a lo largo de todo el experimento, mostró poca variación (alrededor de 310 g) en el peso corporal 
Consumo de Agua
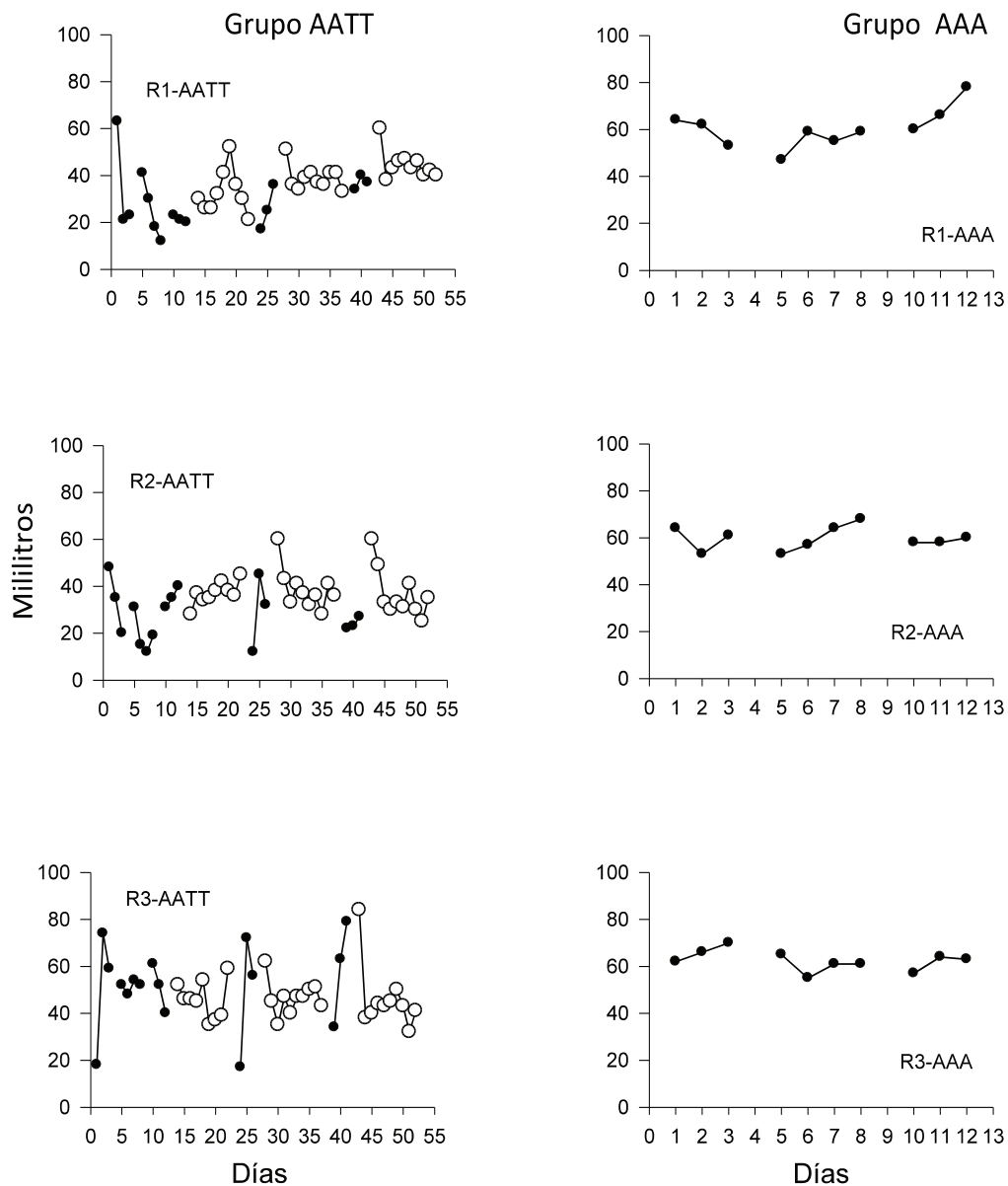

Figura 4. Datos individuales del consumo de agua de los grupos de sujetos AATT (alcohol y agua todo el tiempo) en la columna de la izquierda y AAA (alcohol y agua solo en la inducción) en la columna de la derecha. Los círculos blancos representan los días de libre acceso a la comida. Los círculos negros representan los días iniciales de inducción (solo para el grupo AATT) y de privación de comida para ambos grupos.

durante el periodo de inducción (triángulos negros de la Figura 1). En el primer periodo de libre acceso a la comida el peso corporal incrementó paulatinamente superando los $320 \mathrm{~g}$. En el primer periodo de restricción de la comida el peso corporal disminuyó gradualmente recuperándose durante la siguiente condición de libre acceso a la comida. El segundo ciclo de restricción-libre acceso a la comida prácticamente replicó el patrón mostrado en el primer ciclo. 
El consumo de alimento durante la inducción varió en un rango de entre 12 y $19 \mathrm{~g}$ (triángulos negros de la Figura 2). En el primer periodo de libre acceso a la comida, después de haber consumido $15 \mathrm{~g}$, el consumo incrementó drásticamente en los últimos días del periodo (30 g). Las dos condiciones restantes mostraron un patrón con una tendencia a disminuir el consumo de alimento después de una ingesta mayor a $20 \mathrm{~g}$ en los días iniciales de cada condición.

El consumo de alcohol mostró variaciones durante la inducción (triángulos negros de la Figura 3). En el tercer día ocurrió el consumo de alcohol más alto rebasando los $50 \mathrm{ml}$. El primer periodo de libre acceso de comida y alcohol mostró un consumo inicial alto $(50 \mathrm{ml})$ que disminuyó en el resto de la condición. En los dos periodos de restricción de comida el consumo de alcohol fue mayor que en el periodo de acceso libre previo, aunque el consumo en los dos periodos de acceso libre de comida fue el más bajo de todos los grupos (alrededor de $20 \mathrm{ml}$ ).

Las gráficas individuales (columna izquierda de la Figura 4) muestran que aún estando disponible el agua durante todo el experimento el consumo de agua fue el más afectado. Beber agua fue irregular en todas las fases del experimento, apareciendo mayores consumos de agua el primer día después de las dos restricciones de comida. Aún cuando el bebedero de agua estuvo disponible todo el tiempo durante los periodos de restricción de comida dos sujetos mostraron auto-privación de agua y bebieron más alcohol.

\section{Grupo Alcohol forzado sin inducción (AF)}

Los sujetos de este grupo fueron los que mostraron el menor peso $(280 \mathrm{~g})$ desde el inicio y a lo largo de todo el experimento (triángulos blancos de la Figura 1). El peso corporal fue disminuyendo gradualmente durante la inducción recuperándose hacia el final del primer periodo de acceso libre a la comida. En las restricciones de alimento también hubo una pérdida gradual de peso corporal, siendo más notable el decremento en la primera restricción. El peso corporal incrementó gradualmente durante las condiciones posteriores a la restricción.

El consumo de alimento fue irregular disminuyendo durante los doce días de inducción. Después de ingerir casi $20 \mathrm{~g}$, el consumo se redujo a $6 \mathrm{~g}$ en el tercer día y posteriormente se mantuvo al rededor de $10 \mathrm{~g}$. Este grupo registró los consumos de alimento más bajos en la inducción. En el primer periodo de libre acceso a la comida hubo mayor estabilidad en el consumo alrededor de los $20 \mathrm{~g}$. Aún después de los dos periodos de restricción de alimento el consumo de comida se mantuvo estable con ligeros aumentos hacia la mitad de cada periodo. No se presentó la gran comilona posterior a la restricción de alimento.

El consumo de alcohol se muestra en la Figura 3 (triángulos blancos). Hubo variaciones en un rango de 9-14 g/kg en el consumo de alcohol durante la inducción y el periodo siguiente. La ingesta de alcohol fue la menor de todos los grupos en la primera 
restricción de comida y muy variable en la segunda (5-11 g/ $/ \mathrm{kg})$. D urante los periodos de acceso libre a la comida aunque el consumo fue irregular, alcanzó los consumos más altos de todos lo grupos en estos periodos (al rededor de $15 \mathrm{~g} / \mathrm{kg}$ ).

\section{Discusión}

El objetivo principal de este estudio fue evaluar los efectos de tres procedimientos de inducción al alcohol sobre el mantenimiento del consumo de alcohol, el peso corporal, consumo de comida y de agua en ratas bajo condiciones de restricción de comida, periodos de acceso libre a la comida y disponibilidad de alcohol. Para cumplir con ese propósito se conformaron cuatro grupos de ratas que variaron en la forma en que inicialmente el alcohol fue suministrado.

Los hallazgos principales pueden ser resumidos de la siguiente manera: a) el peso corporal de los sujetos del grupo alcohol forzado con inducción (SA) y del grupo AAA se mantuvo más estable a lo largo del estudio en comparación con los otros dos grupos. El grupo AATT que tuvo disponible agua y alcohol todo el tiempo, presentó el mayor incremento en el peso corporal después de los periodos de restricción de alimento y el grupo alcohol forzado sin inducción (AF) mostró el menor peso corporal a lo largo de todo el experimento; b) el consumo de alimento fue mayor y más variable en el grupo AATT, siendo más homogéneo en el grupo AAA que sólo tuvo el agua y alcohol durante la inducción y similar al grupo SA. El grupo AF mostró el mayor decremento en el consumo de alimento durante el periodo de alcohol inicial sin inducción; c) el consumo de alcohol mostró variabilidad entre grupos durante el periodo de inducción. El grupo SA mostró el mayor consumo en el periodo de inducción y el grupo AAA fue el de menor consumo en este periodo, mientras el grupo AATT mostró el menor consumo de al cohol durante las últimas dos fases de acceso libre a la comida. Con excepción del grupo AATT, el consumo de alcohol se mantuvo estable durante las fases siguientes a la inducción y no hubo una reducción del consumo de alcohol durante los periodos de restricción alimentaria; $y, d$ ) el consumo de agua del grupo AATT, el único que tuvo el agua siempre disponible, mostró efectos de autoprivación y mayores consumos de agua post-privación que los consumos previos a los periodos de restricción de comida; en tanto para el grupo AAA el consumo de agua fue estable durante la inducción al alcohol.

Los resultados de este estudio sugieren que la forma inicial en que se suministra el alcohol puede tener efectos sobre el peso corporal y el consumo de alimento y líquidos en ratas. U na vez conseguido que los sujetos bebieran alcohol evaluamos si la disponibilidad de alcohol en un modelo de auto-administración oral bajo condiciones de restricción-libre acceso a la comida, afectaría el peso corporal y los patrones de consumo de comida, agua y se mantendría el consumo de alcohol de las ratas. Los distintos procedimientos de inducción representan diferentes historias con respecto a los primeros contactos de las ratas con el alcohol, ya sea por beber alcohol forzado en 
una concentración constante (grupo AF alcohol 10\%), o bien incrementado gradualmente la concentración (grupo SA alcohol 4-10\%) constituyen dos formas de contacto inicial con el alcohol. U na historia distinta es que bajo un procedimiento similar al del grupo SA hubiera agua disponible durante la inducción al alcohol (grupo AAA); mientras que el grupo AATT tuvo el agua disponible durante todo el experimento.

En el primer día de la inducción al alcohol los sujetos del grupo SA y del grupo AATT mostraron casi el doble del consumo de alcohol con respecto a los otros dos grupos (AAA y AF). El grupo AAA mantuvo el consumo más bajo de alcohol durante la inducción. Los sujetos del grupo AATT que fueron expuestos al mismo procedimiento de inducción que el grupo AAA, pero que tuvieron alcohol y agua disponible durante todo el experimento, mostraron un mayor consumo de alcohol durante la inducción comparado al del grupo AAA. Sin embargo, el grupo AATT mostró el consumo más bajo de alcohol de todos los grupos en los dos últimos periodos de acceso libre a la comida y fue el que consumió mayores cantidades de alimento en los tres periodos de acceso libre a la comida. Además, en el grupo AATT hubo indicios de autoprivación de agua al disminuir su consumo de agua durante la restricción de alimento y mostrando altos consumos de agua en el día posterior a la restricción de comida comparados con los consumos del día previo a la restricción de comida. Este efecto de autoprivación ya ha sido reportado bajo restricción de alimento pero sin acceso al alcohol (Verplank \& H ayes, 1953).

Los bajos consumos de alcohol del grupo AATT, acompañados de un incremento en el consumo de alimento durante los tres periodos de acceso libre a la comida posteriores a la inducción y la alteración del patrón de beber agua a lo largo del estudio, confirmarían la noción de que los sujetos compensaron su ingesta calórica. U na interpretación podría sugerir que estas alteraciones corresponden con el funcionamiento de mecanismos auto-regulatorios. Desde esta perspectiva, la explicación de la disminución del consumo de alcohol después de la primera restricción de alimento y el aumento de consumo de alimento en las fases de acceso libre a la comida para el grupo AATT, consideraría plausible que sus patrones de consumo tuvieron componentes homeostáticos. Laure-Achagiotis et al (1990) reportaron datos con ratas que muestran una estrecha relación entre comer y beber agua o alcohol. En su estudio para el grupo de ratas que solo tuvo acceso al agua, el $90 \%$ del consumo total de agua estuvo asociado a comer; las ratas bebían agua principalmente durante la comida; en tanto que los dos grupos que solo tuvieron acceso a beber alcohol, cuando bebían lo hacían en un periodo de 20 minutos antes de la comida más que durante 0 posterior a la comida. Las ratas regularon su ingesta de energía y líquidos cuando recibieron soluciones de alcohol (20\%) como única fuente de bebida. Las ratas que bebían alcohol $10 \%$ redujeron hasta un $75 \%$ su ingesta de líquido y un $54 \%$ en ratas que bebían alcohol al 20\% comparadas con el grupo control que solo bebió agua.

Nuestros datos parecen confirmar la relación entre comer y beber alcohol y agua. El grupo AATT que tuvo el agua disponible todo el tiempo fue el que mostró un 
mayor aumento de peso corporal a lo largo del experimento, un mayor consumo de alimento, el menor consumo de alcohol después del periodo de inducción y variaciones en el consumo de agua. Los otros tres grupos mantuvieron un consumo estable de alcohol con ligeras variaciones. Un dato interesante fue que durante los periodos de privación de alimento no aparecieron reducciones en el consumo de alcohol de todos los grupos como ocurre cuando el agua está disponible bajo las condiciones de restricción de alimento. No hubo la auto-privación de alcohol que suele aparecer con la restricción de comida y agua disponible. Sólo el grupo AATT mostró decrementos en el consumo de alcohol en los periodos de libre acceso a la comida. Probablemente debido a que el alcohol es un nutriente y proporciona calorías suficientes para mantener activo a un organismo, ante las condiciones de restricción de alimento las ratas mantuvieron el consumo de alcohol obteniendo de esta manera las calorías necesarias para su equilibrio energético.

El alcohol es una sustancia que pudiera considerarse un macronutriente por la gran cantidad de calorías que aporta, al rededor de $7 \mathrm{kcal} / \mathrm{gr}$ (Yeomans, 2004) y se ha demostrado que en pequeñas cantidades aumenta la ingesta de alimento (Yeomans, Caton, \& Hetherington, 2003). Richter (1926) fue un pionero en reconocer el valor nutriente del alcohol. Demostró que el consumo de alimento en ratas se redujo dependiendo de las calorías que ganaban bebiendo alcohol. Más tarde, Richter (1953) señaló que las calorías del alcohol pueden ser usadas por el organismo de la misma manera como las calorías provenientes de la comida regular. Estudios posteriores han confirmado que el alcohol tiene las calorías suficientes para ser considerado un nutriente importante en la dieta de un organismo (Egli, 2003; Yeomans, 2004; Yeomans et al.,2003). Richter (1941) reportó que las ratas prefieren alcohol diluido en concentraciones mayores del $6 \%$ en lugar de agua, pero beben poco en altas concentraciones de alcohol. Si el alcohol diluido es la única fuente de líquido disponible, las ratas beben sólo para hidratarse y no suele producir efectos patológicos. Los efectos tóxicos del alcohol dependen sobre su concentración en tejidos vulnerables y sobre la acumulación de metabolitos tóxicos, los que a su vez dependen de las tasas de ingestión, distribución y eliminación (D ole \& G entry, 1984). El alcohol no es la primera elección en pruebas de preferencia en ratas (Juárez \& Barrios-D e-Tomasi, 1998), sin embargo, la privación parcial de alimento parece incrementar la preferencia por el consumo de alcohol (Soderpalm \& Hansen, 1999; Spanagel \& Holter, 1999).

A pesar de que en nuestro estudio las ratas de los cuatro grupos estuvieron expuestas a dos periodos de privación total de comida, no se obtuvieron incrementos sostenidos en el consumo de alcohol; sin embargo, los sujetos del grupo que tuvo acceso al agua y al alcohol (AATT) posterior a la inducción no mostraron preferencia por el alcohol. Por el contrario, este grupo bajó su consumo de alcohol y se produjeron alteraciones en el patrón de bebida de agua, confirmando lo reportado por Holloway et al. (1984). Este grupo fue el que mostró los cambios más notables en sus patrones de consumo de alimento, alcohol, agua y peso corporal. Laure-Achagiotis et al. 
(1990) encontraron que las ratas que consumieron alcohol forzado al 20\% ganaron menos peso corporal en comparación con ratas que bebieron alcohol forzado al $10 \%$ y ratas que bebían agua. Los autores confirmaron que el consumo de alcohol causó una notable reducción en la ganancia de peso sin afectar el consumo de comida. Las ratas bebiendo alcohol ajustaron su gasto energético inmediatamente en los ciclos de 24 horas con respecto a la cantidad de calorías proporcionadas por la ingesta de comida. En nuestro caso sólo las ratas del grupo AATT que tuvieron acceso al agua y al alcohol (10\%) ganaron peso después del periodo de inducción al alcohol y de privación de alimento, ya que consumieron más alimento pero bebieron menos alcohol que los otros grupos.

Una explicación alternativa a la hipótesis homeostática se enfocaría más hacia la consideración de las variables motivacionales para tratar con estas alteraciones y formas de consumo de alimento y líquidos. De especial interés resultó el mantenimiento del consumo de alcohol en todos los grupos a lo largo del experimento aún bajo condiciones de restricciones de alimento. Ningún sujeto de todos los grupos dejó de beber alcohol y aunque tampoco se desarrollaron patrones de consumo que reflejaran beber alcohol en exceso, con excepción del grupo AATT, mantuvieron un consumo de alrededor de $20 \mathrm{ml}$. El mantenimiento de patrones relativamente estables de consumo de alcohol bajo condiciones de restricción de comida es un área de estudio relacionada con los factores motivacionales que intervienen en el consumo de alcohol. Dole \& Gentry (1984) tratando de identificar variables motivacionales relacionadas con beber alcohol, cuestionaban si el consumo de alcohol puede ser considerado como un alimento, o bien, como una droga. Es decir, si este consumo es de índole farmacológico cuando está disociado temporalmente de la necesidad de beber agua, o bien homeostático, cuando el alcohol es consumido acompañando la ingesta de comida. En nuestro estudio no se hicieron registros de los niveles de concentración de alcohol en la sangre, ni tampoco de los periodos del día en que consumían el alcohol, pero los sujetos no mostraron un consumo de alcohol que alcanzaran niveles excesivos y dado que fueron expuestos a periodos de restricción alimentaria sus consumos fueron más del tipo auto-regulatorio o compensatorio que farmacológico o tóxico.

A pesar de no utilizar un procedimiento de solución endulzada para inducir el consumo de alcohol, nuestro estudio demostró que es posible mantener consumos de alcohol relativamente estables, aún bajo ciclos de privación de comida y libre acceso a la comida después de la exposición a los diferentes procedimientos de inducción. No se obtuvieron efectos de auto-privación en el consumo de alcohol durante los periodos de privación de comida. Tampoco se obtuvo una ganancia de peso como podría esperarse estando disponible, además de la comida habitual, una fuente adicional de calorías proporcionadas por el alcohol. Laure-Achagiotis et al. (1990) sin emplear un procedimiento de inducción llevaron a cabo un experimento con disponibilidad agua o alcohol (10\% y $20 \%$ ) y comida pero sin restringir la comida. 
Sólo encontraron una menor ganancia de peso con la concentración de alcohol más alta (20\%); mientras que las ratas que bebieron agua o alcohol al $10 \%$ no mostraron diferencia en la ganancia de peso.

La privación alimentaria parece ser una vía confiable para evaluar los efectos de las restricciones sobre el consumo de alimentos (Corwin \& Buda-levin, 2004; Dimitriou, Rice, \& Corwin, 2000; Epstein, Truesdale, Wojcik, Paluch, \& Raynor, 2002; Kupfermann, Kandel, \& Iversen, 2000; López-Espinoza \& Martínez, 2001, 2004, 2005; M artínez \& Gómez, 2009) y agua (Siegel \& Talantis, 1948; Siegel \& Stuckey, 1947; Skinner, 1936). Extender el uso de esta preparación experimental para la evaluación de nutrientes, sabores, y fármacos puede ser una estrategia útil si se busca asegurar el consumo de estas sustancias, fluidos o comidas distintas a las que proporcionan la dieta normal de ratas bajo confinamiento (Avena, 2010). Como ha sido planteado por M eisch (2001), el empleo de modelos experimentales de auto-administración oral para estudiar los efectos conductuales, motivacionales y farmacológicos han sido de valor en los estudios de laboratorio animal. El estudio del consumo de alcohol crónico o temporal, se vería beneficiado por considerar los procedimientos de inducción para beber alcohol como una forma de optimar el control de las primeras experiencias de las ratas con alcohol asegurando su consumo y así evaluar el desarrollo de su ingesta bajo diferentes manipulaciones experimentales.

\section{Referencias}

Avena, N. M. (2010). The study of food addiction using animal models of binge eating. Appetite, 55, 734-737.

Bell, R. L., Rodd, Z. A., Lumeng, L., Murphy, J. M., \& McBride, W. J. (2006). The alcohol-preferring $\mathrm{P}$ rat and animal models of excessive alcohol drinking. Addiction Biology, 11, 270-288.

Bindra, D. (1947). Water-hoarding in rats. Journal of Comparative and Physiological Psychology, 3(40), 149-156.

Barrios De Tomasi E., \& Juárez, J. (2011). Differential effects of chronic naltrexone treatment on food intake patterns and body weight in rats depend on their food deprivation status. European Journal of Pharmacology, 650, 261-267

Corwin, R. L., \& Buda-Levin, A. (2004). Behavioral models of binge-type eating. Physiology and Behavior, 82(1), 123-130.

Cunningham, C., Fidler, T., \& Hill, K. (2000). Animal models of alcohol's motivational effects. Alcohol Research \& Health, 24(2), 85-92.

Dimitriou, S. G., Rice, H. B., \& Corwin, R. L. (2000). Effects of limited access to a fat option on food intake and body composition in female rats. The International Journal of Eating Disorders, 28(4), 436-445.

Dole, V. P. \& Gentry, R. T. (1984). Toward an analogue of alcoholism in mice: Scale 
factors in the model. Proceedings of the National Academy of Sciences of the United States of America USA, Vol. 81, 543-3546.

Egli, M. (2003). Peptides: their role in excess alcohol drinking and their promise as a therapeutic tool. Physiology \& Behavior, 79, 89-93.

Epstein, L. H., Truesdale, R., Wojcik, A., Paluch, R. A., \& Raynor, H. A. (2002). Effects of deprivation on hedonics and reinforcing value of food. Physiology \& Behavior, 78, 221-227.

Grant, K. A., \& Samson, H. H. (1985). Induction and maintenance of ethanol self-administration without food deprivation in the rat. Psychopharmacology, 86, 475-479.

Hagan, M. M., \& Moss, D. E. (1997). Persistence of binge-eating patterns after a history of restriction with intermittent bouts of re-feeding on palatable food in rats: implications for bulimia nervosa. International Journal of Eating Disorders, 22, 411-420.

Heyser, C. J., Schulteis, G., \& Koob, G. F. (1997). Increased ethanol self-administration after a period of imposed ethanol deprivation in rats trained in a limited access paradigma. Alcoholism, Clinical and Experimental Research, 21, 784-791.

Holloway, F. A, Bird, D. C., \& Devenport, J. A. (1984). Periodic availability: factors affecting alcohol selection in rats. Alcohol, 1, 19-25.

Juárez, J., \& Barrios-De-Tomasi, E. (1998). Sex differences in alcohol drinking patterns during forced and voluntary consumption in rats. Alcohol, 19, 15-22.

Kahn, M. y Stellar, E. (1960). Alcohol preference in normal and anosmic rats. Journal of Comparative and Physiological Psychology, 53, 571-575.

Koob, G. F. (2000). Animal models of craving for ethanol. Addiction, 95(2), S73 - S81.

Kupfermann, I., Kandel, E. R., \& Iversen, S. (2000). Hypothalamus and limbic system: Motivation. En E. R. Kandel (Ed.), Principles of neural science. New York: Mc Graw Hill.

Larue-Achagiotis, L., Poussard, A. M., \& Louis-Sylvestre, J. (1990). Alcohol drinking, food and fluid intakes and body weight gain in rats. Physiology \& behavior. 47(3), 545-548.

Linseman, M. A., \& Harding, S. (1989). Alcohol consumption following bidirectional shifts in body weight in rats. Psychopharmacology, 97, 103-107.

López-Espinoza, A., \& Martínez, H. (2001). Efectos de dos programas de privación parcial sobre el peso corporal y el consumo total de agua y comida en ratas. Acta Comportamentalia, 9(1), 5-17.

López-Espinoza, A., \& Martínez, H. (2004). Cambios del patrón alimentario como efecto de la privación de agua o alimento en ratas en crecimiento. International Journal of Psychology and Psychological Therapy, 4(1), 93-104.

López-Espinoza, A., \& Martínez, H. (2005). Efectos de intervalos variables entre periodos de privación sobre el consumo post-privación de agua y comida en ratas. Revista Mexicana de Análisis de la Conducta, 31(1), 67-84. 
Martínez, H., \& Gómez, I. L. (2009). Modelos experimentales para el estudio de la conducta alimentaria. En E. Matute (Ed.), Cerebro: Conducta y Cognición (pp. 71 107). México: Universidad de Guadalajara.

Meisch, R.A. (1984). Alcohol self-administration by experimental animals. En Smart, R.G., Cappell, H.D., Glaser, F.B. Israel, Y., Kalant, H.; Popham, R.E., Schmidt, W., \& Sellers, E.M., (Eds). Research Advances in Alcohol and Drug Problems. Vol. 8, pp. 23-45, NewYork: Plenum Press.

Meisch, R. (2001). Oral drug self-administration: an overview of laboratory animal studies. Alcohol, 24, 117-128.

Mello, N. K. (1973). A review of methods to induce alcohol addiction in animals. Pharmacology Biochemistry and Behavior, 1, 89-101.

Mello, N. K., \& Mendelson, J. H. (1964). Operant performance by rats for alcohol reinforcement: a comparison of alcohol-preferring and nonpre-ferring animals. Quarterly Journal Studies on Alcohol 25, 226 - 234.

Richter, C. P. (1926). A study of the effect of moderate doses of alcohol on the growth and behavior of the rat. Journal of Experimental Zoology, 44, 397-418.

Richter, C. P. (1941). Alcohol as a food. Quarterly Journal Studies on Alcohol, 1, 650-662.

Richter, C. P. (1953). Alcohol, beer and wine as foods. Quarterly Journal Studies on Alcohol, 14, 525-539.

Samson, H. H. ( 1986). Initiation of ethanol reinforcement using a sucrose-substitution procedure in food- and water-sated rats. Alcoholism, Clinical and Experimental Research, 10, 436-442.

Sclafani, A., \& Gorman, A. N. (1977). Effects of age, sex, and prior body weight on the development of dietary obesity in adult rats. Physiology \& Behavior, 18, 1021-1026.

Siegel, P. S., \& Stuckey, H. L. (1947). The diurnal course of water and food intake in the normal mature rat. Journal of Comparative and Physiological Psychology, 40, 365-370.

Siegel, P. S., \& Talantis, B. S. (1948). Water intake as a function of privation interval when food is withheld. The Journal of Comparative and Physiological Psychology, 43, 62-65.

Sinclair, J. D., \& Senter, R. J. ( 1967). Increased preference for ethanol in rats following alcohol deprivation. Psychonomic Science, 8, 11- 12.

Skinner, B. F. (1936). Thirst as an arbitrary drive. The Journal of General Psychology, $15,205-210$.

Slawecki, C. J., \& Samson, H. H. (1997). Changes in oral ethanol self-administration patterns resulting from ethanol concentration manipulations. Alcoholism: Clinical and Experimental Research, 21, 1144-1149

Soderpalm, A. H., \& Hansen, S. (1999). Alcohol alliesthesia: Food restriction increases 
the palatability of alcohol through a corticosterone-dependent mechanism. Physiology and Behavior, 67 (3), 409-415.

Spanagel, R., \& Holter, S. M. (1999). Long-term alcohol self-administration with repeated alcohol deprivation phases: An animal model of alcoholism? Alcohol and Alcoholism, 34(2), 231-243.

Veale, W., \& Myers, R. (1969). Increased alcohol preference in rats following repeated exposures to alcohol. Psychopharmacology, 15, 361-372.

Verplank, W. S., \& Hayes, J. R. (1953). Eating and drinking as a function of maintenance schedule. Journal of Comparative and Physiological Psychology, 46, 327-333.

Yeomans, M. R. (2004). Effects of alcohol on food and energy intake in human subjects: evidence for passive and active over-consumption of energy. British Journal of Nutrition, 92, 31-34.

Yeomans, M. R., Caton, S., \& Hetherington, M. M. (2003). Alcohol and food intake. Current Opinion in Clinical Nutrition and Metabolic Care, 6(6), 639-644. 